Article

\title{
Suitable Pattern of the Natural Environment of Human Settlements in the Lower Reaches of the Yangtze River
}

\author{
Fan Song ${ }^{\circledR}$, Xiaohua Yang * and Feifei Wu \\ State Key Laboratory of Water Environment Simulation, School of Environment, Beijing Normal University, \\ Beijing 100875, China; 201621180014@mail.bnu.edu.cn (F.S.); wufeifei_bnu@163.com (F.W.) \\ * Correspondence: xiaohuayang@bnu.edu.cn; Tel.: +86-010-5880-6050
}

Received: 27 February 2019; Accepted: 9 April 2019; Published: 12 April 2019

\begin{abstract}
The human settlement environment is the object on which human survival depends. In this study, six single factor suitability models and a comprehensive index model of the human settlement natural environment were established. The six single factor models included topography, hydrology, vegetation, soil, temperature and humidity, and land surface temperature. This study took $1 \mathrm{~km} \times 1 \mathrm{~km}$ as the pixel size and relied on the ArcGIS platform to systematically and quantitatively evaluate the human settlement environment of the lower reaches of the Yangtze river. The results show that: (1) From the evaluation results of single natural elements, the topography of the study area is relatively flat, with a small number of hydraulic erosion areas. Besides, there are significant differences between the north and the south in temperature and humidity, hydrology, vegetation, and land surface temperature. (2) In 2015, the most suitable areas of human settlement environment were mainly distributed in the plains along the Yangtze river, the plain of northern Zhejiang, and the Poyang plain. The most unsuitable areas are mainly distributed in mountainous areas, such as the mountain area of southern Zhejiang and Dabie mountain area. Topography and vegetation are the dominant factors for classification. (3) From the perspective of space, the score of the human settlement natural environment in Shanghai is above the average, and the best among the other provinces is Jiangsu province, while Zhejiang, Jiangxi, and Anhui provinces have little difference. From the perspective of time, the overall level of the suitability in the lower reaches of the Yangtze river has been improved from 2005 to 2015, mainly due to the influence of temperature and humidity index and water resource index.
\end{abstract}

Keywords: human settlement suitability; human settlement natural environment index; GIS; lower reaches of Yangtze River

\section{Introduction}

The human settlement environment is the place where people work, live, and play. It is also the dependent object of human survival and the main transformation object of human activities [1]. The quality of the human settlement environment not only directly affects the residents' living experience, but also indirectly affects the economic and social progress of a region. The earliest research on the human settlement environment was in 1958, when the Greek scholar Doxiadis established the science of human settlement and conducted a lot of basic research on the problems of the human settlement environment [2]. The second Habitat Conference of United Nations in the 1990s put forward the "Habitat Agenda"; there were two important issues: "comfortable housing for everyone" and "sustainable development of human settlements in the process of urbanization". This indicated that sustainable human settlements should be established in a human living environment so that 
human beings can enjoy a harmonious, healthy, and fulfilling life with nature. In the 21st century, the concept of sustainable development has become the guiding ideology of the research on the human settlement environment. In recent years, many international scholars have done a lot of research on the sustainable development of a human settlement environment from multiple perspectives. For example, Muhammad Rusdi et al. [3] assessed the land suitability of the Banda Aceh, Indonesia region 10 years after the tsunami. The study was conducted primarily from the perspective of soil permeability, topography, and geology, so as to determine the level of suitability of the area for human habitat. Safa Mazahreh et al. [4] made a land suitability map in Jordan through an approach that integrated soil and climatic data. The analyses not only identified key factors limiting land use but also provided the type of land use for which the region was suitable. Walter Musakwa [5] and Ingrida Bagdanavičiutè [6], respectively, established evaluation standards from the perspectives of nature-society and pollution sources, and then selected the most suitable areas for human settlement in their respective study areas, so as to provide a basis for land reform and creation of smart cities. Although their researches have their own emphases, they all make great contributions to the study of human settlement.

In China, the research on the human settlement environment started in the 1990s. Tsinghua University established the research center for human settlements in 1995. Professor Wu Liang-yong classified the human settlement environment system into five systems: natural system, human system, social system, residential system, and supporting system [7,8]. Around these five systems, scholars in different fields have conducted a lot of studies from different perspectives. Scholars in the field of architecture are more focused on the study of the residential system and support system, among the five major systems, while sociologists are more concerned with human systems and social systems [9], and environmental and ecologists are more inclined to study natural systems. There are also some scholars who study complex systems [10]. A review article by MA Ren-feng gave a detailed description of the history and current situation of research on the human settlement environment in China [11], and the evaluation method of human settlement natural environment suitability, introduced in the article, is also the main idea of this paper.

In addition to the system divisions described above, the research on the human settlement environment can also be divided from the spatial scale, which can be divided into five scales including global, regional, urban, community, and architecture [12]. Numerous classifications have also generated numerous research methods. Currently, the commonly used method is analysis in Geographic Information System. At the same time, many mathematical methods such as fuzzy comprehensive evaluation [13], factor analysis [14], and genetic algorithm [15] have been applied in this field. With the development and maturity of Geographic Information System and Remote Sensing technology, it is possible to show the advantages and disadvantages of the human settlement environment with pictures and texts. The joint analysis of GIS tool and other methods is also gradually developed [16], which is of great significance to the research on the human settlement environment suitability.

Natural system is the basic system of the human settlement environment, which directly relates to human health and body surface feeling, and indirectly constitutes the construction foundation of other systems. Among the numerous natural factors, this study selects some basic elements that are most closely related to human survival and life, including six natural elements: topography, hydrology, vegetation, soil, temperature and humidity, and land surface temperature. On the basis of the study of each individual element, the model of the human settlement natural environment index (HNEI) is established, and different weights are assigned to each element according to the actual situation of the research area. The lower reaches of the Yangtze river are one of the most developed regions in China. During a long period of economic development, a series of resource and environmental problems have emerged. The purpose of this study is as follows: 1 . To reveal the spatial pattern of the human settlement natural environment suitability of five provinces in the lower reaches of the Yangtze river; 2. to explore the advantage and restriction of the human settlement environment in this region, so as to 
provide the corresponding research basis and decision-making support for local population spatial planning and land function planning.

\section{Study Area}

The study area covered the lower reaches of the Yangtze river, including five provincial-level administrative regions of Jiangxi, Anhui, Jiangsu, Zhejiang and Shanghai, with a total of 51 prefecture-level cities and one municipality directly under the central government. The region is located in longitude $\mathrm{E} 113^{\circ} 34^{\prime}-\mathrm{E} 123^{\circ} 25^{\prime}$ and latitude $\mathrm{N} 24^{\circ} 29^{\prime}-\mathrm{N} 35^{\circ} 20^{\prime}$. The total area is about $526,080 \mathrm{~km}^{2}$. The location of the study area is shown in Figure 1. At the end of 2016, the permanent resident population of the five provinces was about 267.82 million, accounting for $19.37 \%$ of the total population of China, with a high population density. Most of the region is located in the economic radiation zone of the urban agglomeration of the Yangtze river delta. Relying on the excellent geographical advantages and economic foundation, the study area has become the region with the highest urbanization level and the most developed economy in China. Most of the region belongs to the subtropical monsoon climate zone, with obvious monsoon characteristics. In the process of rapid urbanization in this region, a lot of resources and environmental problems have been caused, resulting in great changes in the natural environment. In this study, the grid of $1 \times 1 \mathrm{~km}$ was taken as the basic unit, which can satisfy the analysis of different spatial scales, so as to make an appropriate evaluation of the natural suitability of human settlement.

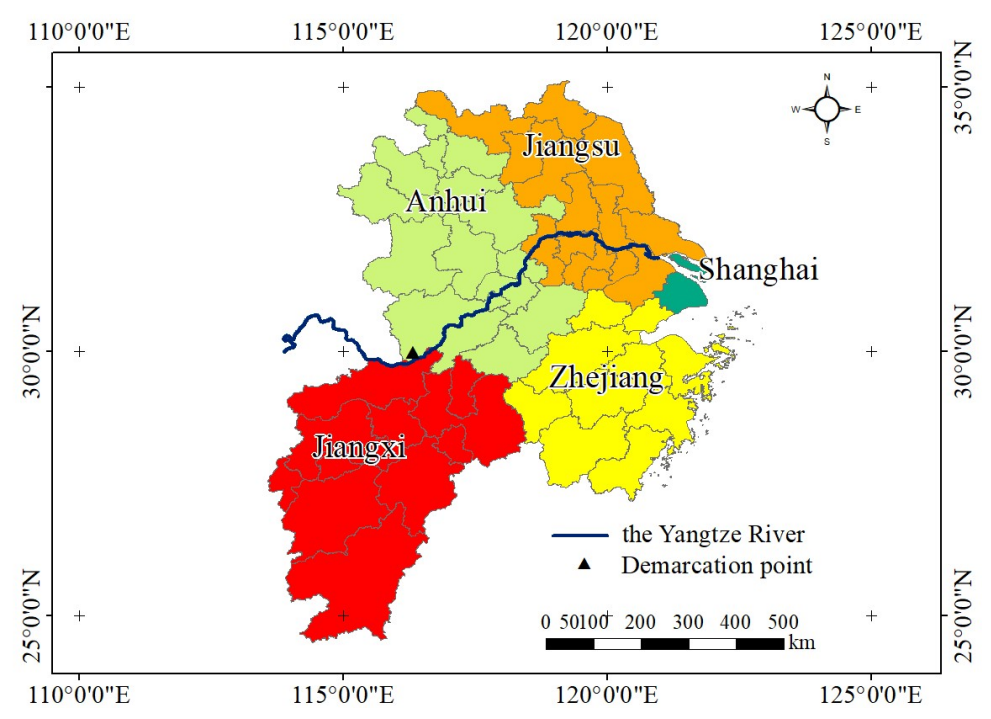

Figure 1. Location of study area.

\section{Materials and Methods}

\subsection{Data Sources}

The six indexes selected in this study were: relief degree of land surface (RDLS), water resource index (WRI), land cover index (LCI), soil suitability index (SSI), temperature-humidity index (THI), and land surface temperature index (LSTI). The data used for calculation included: digital elevation model data, land use data, annual precipitation spatial interpolation data, normalized difference vegetation index data [17], soil erosion spatial distribution data [18], annual data for natural indicators of weather stations, and MODIS land surface temperature data. The data types, year of data, and data source are shown in the Table 1. 
Table 1. Details of the data.

\begin{tabular}{|c|c|c|c|}
\hline The Name of the Data & Data Types & Year of Data & Data Source \\
\hline Digital elevation model data & Raster data, $90 \times 90 \mathrm{~m}$ & 2015 & $\begin{array}{l}\text { The resource and environmental data cloud } \\
\text { platform of the Chinese academy of sciences } \\
\text { (http://www.resdc.cn/) }\end{array}$ \\
\hline Land use data & Raster data, $1 \times 1 \mathrm{~km}$ & $2005,2010,2015$ & $\begin{array}{l}\text { The resource and environmental data cloud } \\
\text { platform of the Chinese academy of sciences } \\
\text { (http://www.resdc.cn/) }\end{array}$ \\
\hline $\begin{array}{l}\text { Annual precipitation spatial } \\
\text { interpolation data }\end{array}$ & Raster data, $1 \times 1 \mathrm{~km}$ & 2004-2006,2009-2011,2014-2016 & $\begin{array}{l}\text { The resource and environmental data cloud } \\
\text { platform of the Chinese academy of sciences } \\
\text { (http://www.resdc.cn/) }\end{array}$ \\
\hline $\begin{array}{l}\text { Normalized difference vegetation } \\
\text { index data }\end{array}$ & Raster data, $1 \times 1 \mathrm{~km}$ & $2005,2010,2015$ & $\begin{array}{l}\text { The resource and environmental data cloud } \\
\text { platform of the Chinese academy of sciences } \\
\text { (http://www.resdc.cn/) }\end{array}$ \\
\hline Soil erosion spatial distribution data & Raster data, $1 \times 1 \mathrm{~km}$ & 2015 & $\begin{array}{l}\text { The resource and environmental data cloud } \\
\text { platform of the Chinese academy of sciences } \\
\text { (http://www.resdc.cn/) }\end{array}$ \\
\hline $\begin{array}{l}\text { Annual data for natural indicators of } \\
\text { weather stations }\end{array}$ & Point data & 2004-2006,2009-2011,2014-2016 & $\begin{array}{l}\text { Chinese academy of sciences } \\
\text { (http://www.resdc.cn/) }\end{array}$ \\
\hline MODIS land surface temperature data & Raster data, $1 \times 1 \mathrm{~km}$ & $2005,2010,2015$ & $\begin{array}{l}\text { United States Atmosphere Archive \& Distribution } \\
\text { System, Distributed Active Archive Center } \\
\text { (https://ladsweb.modaps.eosdis.nasa.gov/). }\end{array}$ \\
\hline
\end{tabular}




\subsection{Methods of Single Factor Model}

\subsubsection{Relief Degree of Land Surface}

Relief degree of land surface refers to the difference between the highest and lowest elevations in an area. It reflects the macroscopic changes of the region's terrain, which is of great reference value for the site selection of human settlements [19]. The key to calculate RDLS is to determine the size of the reference area, which can well express the topographic characteristics of the study area. Referring to the research results of Feng Zhi-ming [20], Wang Yong-li [21], and other scholars, the formula of RDLS established in this paper is as follows:

$$
R D L S=A L T / 1000+\{[\max (\mathrm{H})-\min (\mathrm{H})] \times[1-\mathrm{P}(\mathrm{A}) / \mathrm{A}]\} / 500
$$

where, ALT refers to the average altitude within the selected reference area; $\operatorname{Max}(\mathrm{H})$ and $\min (\mathrm{H})$ refer to the highest and lowest elevations within the reference area; $\mathrm{P}(\mathrm{A})$ refers to the flat area within the reference area, whose slope is less than $5^{\circ}$; A refers to the total area of the reference area; and 500 refers to China's benchmark mountain height.

The neighborhood analysis method based on raster data was used to calculate the size of the reference area [22]. The neighborhood analysis tool of ArcGIS was used to analyze the altitude difference within the grid, from $3 \times 3$ pixels to $69 \times 69$ pixels. According to the calculation results, the scatter diagram of the corresponding relationship between the grid size and the altitude difference was established, and the logarithmic function of the curve equation was determined. The size of the reference area grid can be determined according to the change of curve growth rate. In this study, the determined optimal grid size was $19 \times 19\left(2.92 \mathrm{~km}^{2}\right)$. According to Formula (1), the RDLS of the lower reaches of the Yangtze river can be obtained. The result in 2015 is shown in Figure 3a.

\subsubsection{Water Resource Index}

Water is the source of life, and the basic need of human settlement construction and development. Although the lower reaches of the Yangtze river are relatively rich in water resources, they are unevenly distributed. In order to express this difference, referred to the environmental assessment standards of the ministry of environmental protection of the People's Republic of China, and relevant research results $[23,24]$, we selected two indexes of "rainfall" and "distance from water source" to build the water resource index model. The formula of WRI is as follows:

$$
W R I=\alpha \mathrm{P}+\beta \mathrm{D}
$$

where $\mathrm{P}$ is the normalized rainfall; $\mathrm{D}$ is the normalized distance from the water source; $\mathrm{A}$ and $\mathrm{B}$ are the weights of two factors, which are respectively 0.8 and 0.2 in this study. In order to reduce the contingency of data, the average rainfall in 2004-2006, 2009-2011, and 2014-2016 was used as the values in 2005, 2010, and 2015, respectively. The required water source location was obtained by extracting the "water area" type in the land use data and the "Euclidean Distance" in ArcGIS was used to calculate the "distance from water source". In this paper, the distance within $10 \mathrm{~km}$ from the water source was calculated and reversely normalized, and the value of the remaining areas was assigned to zero. The calculation result in 2015 is shown in Figure 3b.

\subsubsection{Land Cover Index}

Land is an important material for human survival and development. Land coverage and utilization not only affect the stability of natural ecosystem, but also continuously affect the development of social economy [25]. In recent years, with the rapid development of urbanization, inappropriate land use has become more and more serious. Inappropriate land use has become a problem of universal concern, among which vegetation cover has been a hot topic. Vegetation is an important intermediate link 
connecting the atmospheric environment, soil environment, and water environment, which can regulate the climate and have a significant impact on the human settlement environment [26]. In this paper, the land cover index model was constructed by referring to the ecological environment evaluation standard of the State Environmental Protection Administration [27] and previous research results [24]:

$$
L C I=L T_{i} \times N D V I
$$

where, $L T_{i}$ refers to the weight of each land use type, and NDVI refers to the normalized difference vegetation index.

According to the research purpose, relevant literatures [28,29] and technical specifications, weight was assigned to each land use type, and the results are shown in Table 2. Then, the land cover index can be obtained according to Formula (3). The calculation result in 2015 is shown in Figure 3c.

Table 2. Weight of each land use type.

\begin{tabular}{cccc}
\hline Land-Use Type & Weight & Classification & Weight \\
\hline Arable land & 0.25 & Paddy field & 0.65 \\
& & Dry field & 0.35 \\
Woodland & 0.18 & Forest land & 0.34 \\
& & Shrub land & 0.26 \\
& & Sparse wood & 0.2 \\
Grass land & \multirow{2}{*}{0.15} & Other woodland & 0.2 \\
& & Grassland with high coverage & 0.4 \\
Water area & \multirow{2}{*}{0.17} & Grand with moderate coverage & 0.33 \\
& & Crand with low coverage & 0.27 \\
& & Lake & 0.31 \\
& & Reservoir and pit-pond & 0.29 \\
Construction land & \multirow{2}{*}{0.2} & Mudflat & 0.2 \\
& & Bottomland & 0.1 \\
& & Urban and town land & 0.1 \\
Unused land & \multirow{2}{*}{0.05} & Village land & 0.44 \\
& & Other construction land & 0.35 \\
& & Wetland & 0.21 \\
& & Bare land & 0.48 \\
& & Bare rock & 0.28 \\
& & & 0.24 \\
\hline
\end{tabular}

\subsubsection{Soil Suitability Index}

Soil erosion is a serious environmental problem, which not only damages land resources and affects normal industrial and agricultural behaviors, but also may aggravate drought and waterlogging. It is one of the important evaluation indexes of the human settlement environment [30]. China is one of the countries with serious soil erosion, which has affected the economic and social development. It can be known from the existing studies that soil erosion exists in some areas of the study area, especially in Jiangxi province [31,32]. Therefore, soil erosion was taken as an evaluation index in this study. We used soil erosion data for reclassification to obtain soil suitability index of five provinces in the lower reaches of the Yangtze river, as shown in Figure 3d.

\subsubsection{Temperature-Humidity Index}

Among the many climatic elements, air temperature directly affects the exchange of heat and water between human body and the outside world, so the air temperature directly affects the comfort degree of human body. Due to the difference in humidity between the north and the south, there will be a difference in apparent temperature [33]. Therefore, this study adopted the widely used temperature-humidity index (THI) to express this effect [34,35], so as to reflect the temperature 
suitability through the comprehensive effect of temperature and humidity. The calculation formula is as follows:

$$
\begin{aligned}
& \mathrm{THI}=\mathrm{T}-0.55(1-f)(\mathrm{T}-58) \\
& \mathrm{T}=1.8 \mathrm{t}+32
\end{aligned}
$$

where $\mathrm{t}$ is Celsius, $\mathrm{T}$ is Fahrenheit, and $f$ is relative humidity. In order to reduce the contingency of data, the average temperature and relative humidity data in 2004-2006, 2009-2011, and 2014-2016 was used as the values in 2005, 2010, and 2015, respectively. Firstly, the location of weather stations in the study area and the corresponding temperature and humidity information were input in ArcGIS. The locations of weather stations are shown in Figure 2. After data processing, the kriging interpolating model was used to carry out spatial interpolation processing in five provinces, and the spatial distribution data of various climatic elements were obtained. We mainly used the "Spatial Analyst tools-interpolation-Kriging" function in ArcGIS. Finally, according to Formula (4), the 2015 result is shown in Figure 3e.

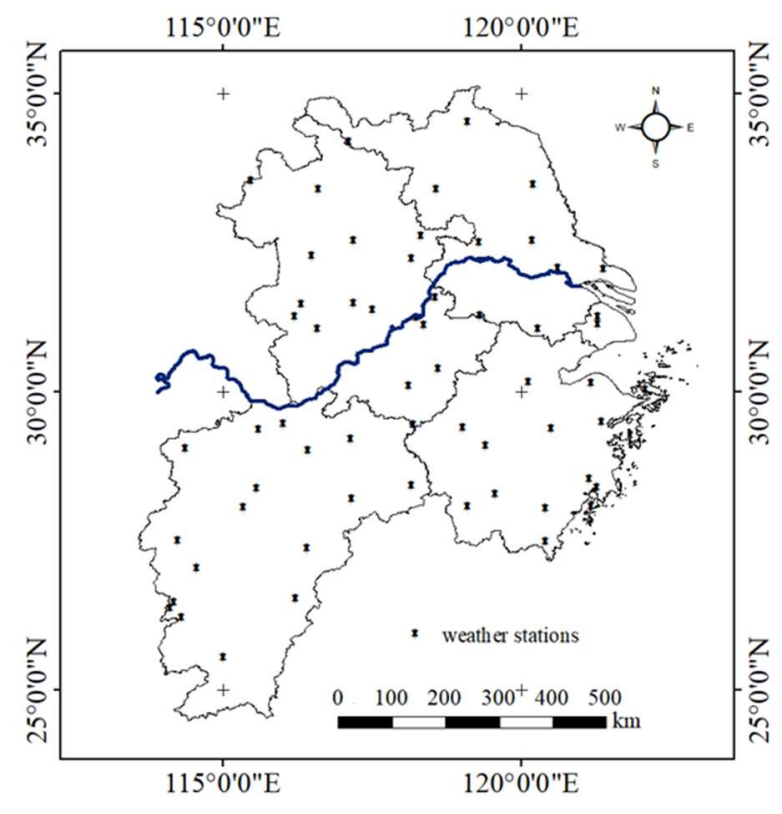

Figure 2. Locations of weather stations.

\subsubsection{Land Surface Temperature Index}

The rapid development of urbanization has changed the urban climate, and one of the remarkable characteristics is the urban heat island effect. On the one hand, the urban heat island effect directly affects the energy utilization, hydrological environment, and air quality. On the other hand, the urban heat island effect also indirectly affects the quality of urban living environment and the health status of residents [36]. Land surface temperature is a key parameter of urban surface energy balance and a very important parameter for monitoring urban heat island effect.

The classification method of land surface temperature was to segment the temperature density on the basis of the original land surface temperature data [37]. The surface temperature in the region was divided into five grades, which were: low temperature $\left(\mathrm{T}<\mathrm{T}_{\text {mean }}-1.5 \mathrm{~T}_{\mathrm{std}}\right)$; sub-low temperature $\left(\mathrm{T}_{\text {mean }}-1.5 \mathrm{~T}_{\text {std }}<\mathrm{T}<\mathrm{T}_{\text {mean }}-0.5 \mathrm{~T}_{\text {std }}\right)$; medium temperature $\left(\mathrm{T}_{\text {mean }}-0.5 \mathrm{~T}_{\text {std }}<\mathrm{T}<\mathrm{T}_{\text {mean }}+0.5 \mathrm{~T}_{\text {std }}\right)$; sub-high temperature $\left(\mathrm{T}_{\text {mean }}+0.5 \mathrm{~T}_{\text {std }}<\mathrm{T}<\mathrm{T}_{\text {mean }}+1.5 \mathrm{~T}_{\text {std }}\right)$; high temperature $\left(\mathrm{T}>\mathrm{T}_{\text {mean }}+1.5 \mathrm{~T}_{\text {std }}\right)$. Where $T_{\text {mean }}$ is the average value of land surface temperature and $T_{\text {std }}$ is the standard deviation of land surface temperature. The result of land surface temperature index in 2015 is shown in Figure 3f. 


\subsection{Methods of Human Settlement Natural Environment Model}

\subsubsection{Scoring Criteria}

After the completion of the single factor evaluation, it is necessary to carry out the graph superposition to complete the evaluation of human settlement natural environment. Therefore, each single factor model should be graded according to the actual situation of the study area and existing studies [38]. The single factor evaluation results in 2015 were divided into five grades and assigned with scoring values, as shown in Table 3.

Table 3. Scoring criteria of the single factors.

\begin{tabular}{cccc}
\hline & RDLS & WRI & LCI \\
\hline I (90 grades) & {$[-0.02,0.15]$} & $(0.67,0.96]$ & $(0.1209,0.1495]$ \\
II (70 grades) & $(0.15,0.44]$ & $(0.54,0.67]$ & $(0.0807,0.1209]$ \\
III (50 grades) & $(0.44,1.03]$ & $(0.41,0.54]$ & $(0.0562,0.0807]$ \\
IV (30 grades) & $(1.03,1.68]$ & $(0.25,0.41]$ & $(0.0323,0.0562]$ \\
V (10 grades) & $(1.68,3.30]$ & {$[0,0.25]$} & {$[-0.004,0.0323]$} \\
\hline & SSI & THI & LSTI \\
\hline I (90 grades) & mired & $(61,63]$ & low temperature \\
II (70 grades) & mild & $(59,61],(63,66]$ & sub-low temperature \\
III (50 grades) & moderate & $(57,59]$ & medium temperature \\
IV (30 grades) & strong & $(55,57]$ & sub-high temperature \\
V (10 grades) & very strong and violent & {$[46,55]$} & high temperature \\
\hline
\end{tabular}

Note: RDLS refers to Relief Degree of Land Surface; WRI refers to Water Resource Index; LCI refers to Land Cover Index; SSI refers to Soil Suitability Index; THI refers to Temperature-Humidity Index; LSTI refers to Land Surface Temperature Index.

\subsubsection{Establishment of Human Settlement Natural Environment Index}

On the basis of single factor evaluation, a human settlement natural environment index (HNEI) model was constructed to evaluate the natural suitability of human settlement in five provinces of the lower reaches of the Yangtze river. The formula is as follows:

$$
H N E I=\alpha R D L S+\beta W R I+\chi L C I+\delta S S I+\gamma T H I+\varepsilon L S T I+0.2 G D P I
$$

where $\alpha \beta \chi \delta \gamma \varepsilon$ are the corresponding weights of each index respectively, which were obtained by referring to existing studies $[4,28,38]$ through the analytic hierarchy process. The results were as follows: $\alpha=0.2324, \beta=0.0794, \chi=0.3579, \delta=0.0435, \gamma=0.1697, \varepsilon=0.1172$.

0.2GDPI (Gross Domestic Product Index) is the economic correction coefficient. Since selected natural indexes such as LCI and LSTI are affected largely by population distribution, the economic coefficient was added to eliminate such influence. At the same time, economically developed areas have relatively complete residential infrastructure, which is also one of the necessary conditions for human settlement. The classification of GDPI is shown in Table 4. 
Table 4. The classification of Gross Domestic Product Index (GDPI).

\begin{tabular}{cc}
\hline Grades & Classification of GDP (unit: Yuan per $\mathbf{~ k m}^{\mathbf{2}}$ ) \\
\hline 10 & {$[0,424]$} \\
20 & $(425,558]$ \\
30 & $(558,983]$ \\
40 & $(983,2337]$ \\
50 & $(2337,6654]$ \\
60 & $(6654,20,415]$ \\
70 & $(20,415,64,282]$ \\
80 & $(64,282,204,114]$ \\
90 & $(204,114,649,851]$ \\
100 & $(649,851,2,070,711]$ \\
\hline
\end{tabular}

\section{Results and Discussion}

\subsection{Results of Single Natural Factor}

(1) RDLS: As can be seen from Figure 3a, the RDLS in most areas was low, with the maximum of 3.3 and the minimum of -0.03 . From the perspective of province, there were some high-RDLS areas in Anhui, Zhejiang, and Jiangxi provinces, which were distributed in the Dabie Mountain area, mountainous area of southern Anhui, mountain area of southern Zhejiang, and the Mufu Mountain, Jiuling Mountain in northwest Jiangxi province.

(2) WRI: As can be seen from Figure 3b, the WRI showed a trend of gradual decrease from south to north, with high suitability areas concentrated in central and northern Jiangxi, southern Anhui, and most areas of southwest Zhejiang. The low suitability areas were mainly concentrated in the central and northern Anhui and most areas of Jiangsu province.

(3) LCI: As shown in Figure 3c, the LCI had the following characteristics: 1. In terms of spatial distribution, a medium-high-low pattern was formed from north to south. The dominant vegetation types in the three regions were dry field, paddy field, and woodland, respectively. 2 . In terms of provinces, Jiangsu province had the best suitability. Except for the uninhabitable lake areas, the scores of the other regions were all above the average. The LCI of Anhui from north to south also presented a medium-high-low distribution. Because the built-up areas in Shanghai were too concentrated, the LCI of the northern Shanghai was poor. The most suitable areas of Jiangxi and Zhejiang were the areas of basins and plains.

(4) SSI: As can be seen from Figure 3d, soil erosion in the lower reaches of the Yangtze river was only caused by hydraulic erosion without wind erosion and freeze-thaw erosion, which was related to the geographical location of the region. The numerous tributaries along the Yangtze river and the abundant rainfall contributed to this phenomenon.

(5) THI: According to Figure 3e and the reference standard, the THI of the lower reaches of the Yangtze river was suitable for human habitation. From the perspective of distribution, the THI basically increased with the increase of latitude. The overall temperature-humidity index of Jiangxi province was the highest, while that of Anhui province and Jiangsu province was lower. In addition, the THI of mountainous areas was obviously lower than that of plain areas at the same latitude.

(6) LSTI: According to the actual situation of the research area, we believed that the lower the land surface temperature is, the better the habitability is. Figure $3 \mathrm{f}$ shows that Shanghai had the highest land surface temperature among the five provinces, because Shanghai had a high population density and a high proportion of built-up area. Due to the existence of a large number of lakes, such as Tai Lake and Hongze Lake, Jiangsu province had reserved a large area of low temperature area, while the high temperature area was mostly concentrated in the urban agglomeration in southern Jiangsu. There was a close relationship between land surface temperature and topography in Zhejiang and Anhui provinces. High temperature areas were mainly concentrated in plain and basin areas; low 
temperatures areas were concentrated in hilly and mountainous areas. Jiangxi province is located in the south of the five provinces, the sun is the strongest, so the land surface temperature was relatively high.
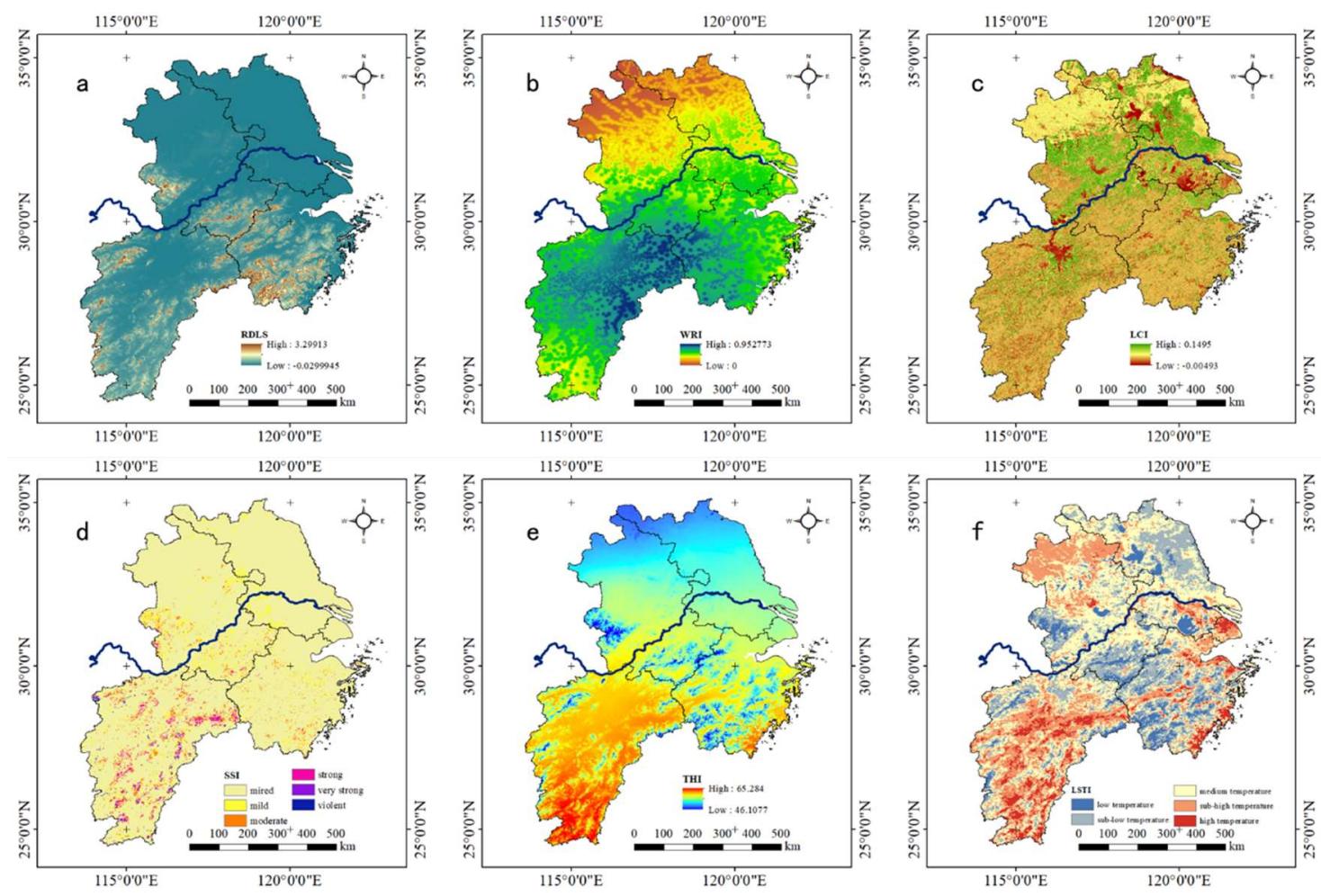

Figure 3. Suitability evaluation of single natural elements. (a) RDLS; (b) WRI; (c) LCI; (d) SSI; (e) THI; (f) LSTI.

\subsection{Results of Human Settlement Natural Environment}

The distribution diagram of Human Settlement Natural Environment Index (HNEI) obtained after superposition calculation is shown in Figure $4 \mathrm{a}$. It can be seen from the figure that the range of HNEI was 22.2-99.3. The results are expressed in five levels respectively: very unsuitable $(22 \leq \mathrm{HNEI} \leq 48)$, unsuitable $(48<\mathrm{HNEI} \leq 57)$, medium suitable $(57<\mathrm{HNEI} \leq 66)$, suitable $(66<\mathrm{HNEI} \leq 77)$, and very suitable $(77<\mathrm{HNEI} \leq 100)$.
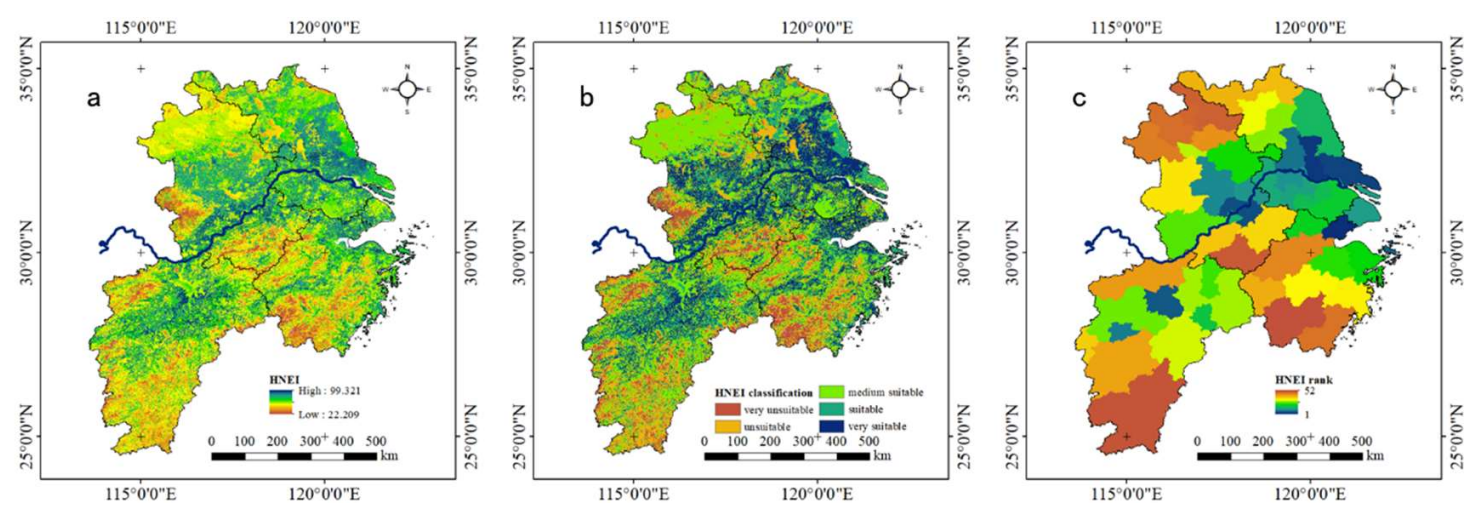

Figure 4. Evaluation results of Human Settlement Natural Environment Index (HNEI). (a) HNEI; (b) HNEI classification; (c) HNEI rank.

As can be seen from Figure $4 b$, the regions with high HNEI were mainly distributed along the Yangtze river, the northern Zhejiang plain, and the Poyang lake plain. The specific analysis is as follows: 
(1) Very unsuitable area: This area was the most unsuitable area for human habitation in the study area, which was not suitable for development and had a fragile ecological environment. This area accounted for $7.27 \%$ of the total area. As can be seen from the figure, this part of the region was mainly distributed in southern Zhejiang mountain, Dabie mountain, and southern Anhui mountain. Besides, there was Mufu mountain and Jiuling mountain in northwest Jiangxi province. The dominant factor in these areas was RDLS. In addition, these areas also tended to be consistent in other single factor evaluation results. As for the LCI, most of these areas were covered by forest, and the scoring criteria was IV (30 grades). In terms of LSTI, due to the high vegetation coverage, most scoring criteria in these areas were II (70 grades) or above. In terms of THI, due to the high terrain, the somatosensory temperature in these areas was relatively cold, and most of the scoring criteria were III (50 grades) or below. Finally, in addition to the southern Zhejiang mountains, other areas had varying degrees of erosion.

(2) Unsuitable area: This area accounted for $20.14 \%$ of the total area and was widely distributed. The reasons for the formation of such areas were complex and can be roughly divided into five categories: The first category was the surrounding area of the very unsuitable area. The terrain and other factors in this area were better than those in the very unsuitable area. The second category was distributed in mountainous and hilly areas in southern Jiangxi. The terrain of this category was between mountain and plain, and the score of RDLS increased, but correspondingly, the score of LSTI decreased. The WRI of this area differed widely, there was less rainfall in southern Jiangxi and more in eastern Jiangxi. The biggest difference from the first category was in the THI. Such a category had lower latitudes and higher temperatures, making it more suitable for settlement. The third category was the western Zhejiang hilly region and the eastern Zhejiang hilly region. Similar to the first category, the dominant factors were RDLS and LCI. In general, this category was limited by THI, so the natural ecological conditions were slightly worse than those of the second category. However, the GDP of this area was higher than that of the second category; there was a strong ability to construct and transform the residential environment. After correction, they were all in the unsuitable area. The fourth category was located in the lake area of northern Anhui and northern Jiangsu. The water area itself was uninhabitable and the temperature was low, so it was in the unsuitable area. The fifth category was the northern region of the study area. The limiting factor in this category was the THI, and the temperature was relatively cold.

(3) Very suitable area: This area had the most harmonious combination of various natural factors, and was the most suitable place for human production and life. This category accounted for $21.19 \%$ of the total area, mostly distributed in the plain areas along the Yangtze river, the plain areas in northern Zhejiang, and the Poyang lake plain. There were two dominant factors in this area. The first one was LCI, with scores of 70 or above. The vegetation coverage in these regions was basically paddy fields, which were suitable for living. The second was RDLS. These areas were all flat and suitable for large-scale group living.

(4) Suitable area: This category was also suitable for human habitation, accounting for $16.91 \%$ of the total area. This area was relatively complex, which can be roughly divided into three categories: The first category was the area around the Yangtze river basin, which was distributed around the very suitable area. The dominant factor in this region was the LCI, and most of the vegetation was paddy field. The second category was parts of Nantong city, Yancheng city, and part of northern Jiangsu. The dominant factor of this region was still the LCI, and most of them were dryland regions with relatively good hydrothermal conditions. In addition, the correlation degree between this category and the LSTI was relatively high, and most of them were located in the region with medium temperature. The third category was the area around Poyang lake plain in Jiangxi province and the basin area in the south of Poyang lake. The dominant factors in this part were WRI and RDLS, and most of them were in flat areas and areas with abundant water resources.

(5) Medium suitable area: This category covered the largest area among the five categories, accounting for $34.56 \%$ of the total area, with a large distribution in each province. Generally, there 
were the following distribution areas: The first category was the part of northern Anhui and northern Zhejiang province, the dominant factor was the land cover index, and the land use type was mostly dry land. The limiting factors were the THI and WRI. The temperature in most regions was low and the amount of water resources was small; the score of the two indexes were mostly below 30 points. The second category covered most of Shanghai and some parts of southern Jiangsu province. The dominant factor of this category was LCI, and the limiting factor was LSTI. The main land use type was construction land, and the natural conditions of this region were more suitable for living. However, it was located in the Yangtze river delta region, and the built-up area had a large population density, so the LSTI was higher. In fact, it was no longer suitable for adding too many people, so it was classified in the medium suitable area. The third category was Tai lake, Poyang lake, and other lake areas, which were similar to the lake in unsuitable areas. The fourth category was the area between the suitable areas and the unsuitable areas. If such areas can follow the local development rules and make rational development and utilization in the development process, they are highly likely to become suitable areas. On the contrary, if the ecological environment is damaged or the climate changes greatly, they are also likely to become unsuitable areas.

The ranking of HNEI in 2015 is shown in Figure 4c. Similar to Figure 4b, cities with a high index were mainly distributed along the Yangtze river, the northern Zhejiang plain, and the Poyang lake plain. Shanghai's HNEI ranked twelfth. Among the other four provinces, Jiangsu province had the best HNEI. There were six cities in the top 15: Taizhou, Nantong, Yangzhou, Zhenjiang, Changzhou, and Nanjing. The overall scores of the other three provinces were similar. The top 15 cities in Zhejiang province are Jiaxing and Zhoushan. Jiangxi province had Nanchang, Xinyu. Anhui province had Wuhu, Hefei, Ma'anshan, and Tongling.

\subsection{Time Evolution Analysis of HNEI}

In order to explore the evolution of HNEI in the study area, WRI, LCI, THI, and LSTI in 2010 and 2005 were recalculated using relevant data to obtain the HNEI of 2010 and 2005. The results were graded using the same criteria as in 2015, as shown in Figure 5 and Table 5.
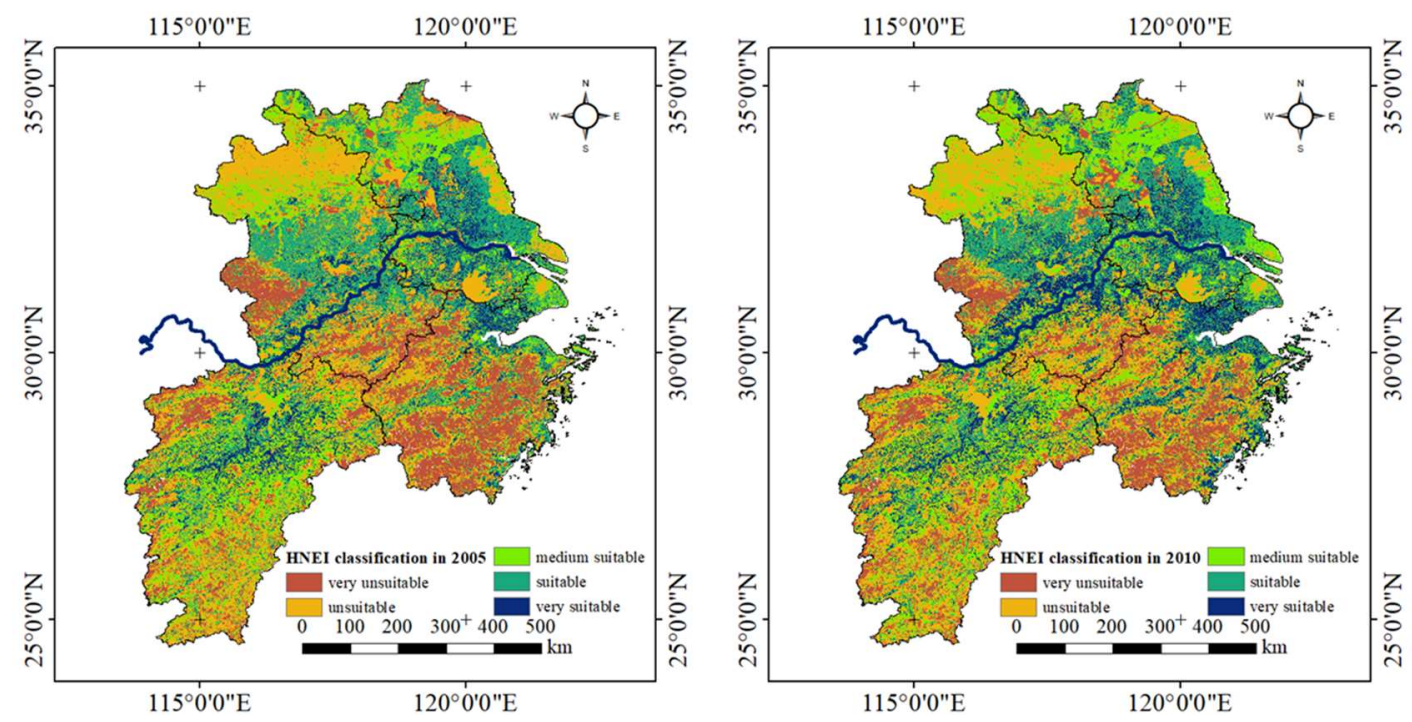

Figure 5. Results of natural suitability of human settlements in 2005 and 2010. HNEI classification in 2005; HNEI classification in 2010.

According to the results in Figure 5 and the result of 2015, the natural suitability of human settlement in the lower reaches of the Yangtze river showed little change, and the high suitability areas were still concentrated along the Yangtze river, the northern Zhejiang plain, and the Poyang lake plain. However, the overall level of HENI was slightly improved; it can be seen from the results in 
Table 5 that the area of very unsuitable was significantly reduced, while the area of very suitable was significantly increased. It was found that the leading factors are THI and WRI, and the improvement of these two indexes increased the overall score, making the natural suitability of human settlement somewhat improved compared with that of ten years ago.

Table 5. The change of area proportion of each district.

\begin{tabular}{cccccc}
\hline & $\begin{array}{c}\text { Very } \\
\text { Unsuitable }\end{array}$ & Unsuitable & $\begin{array}{c}\text { Medium } \\
\text { Suitable }\end{array}$ & Suitable & Very Suitable \\
\hline 2015 & $7.20 \%$ & $20.14 \%$ & $34.56 \%$ & $16.91 \%$ & $21.19 \%$ \\
2010 & $14.30 \%$ & $30.82 \%$ & $22.29 \%$ & $18.31 \%$ & $14.28 \%$ \\
2005 & $17.80 \%$ & $31.93 \%$ & $20.04 \%$ & $20.59 \%$ & $9.64 \%$ \\
\hline
\end{tabular}

\section{Conclusions}

(1) In this paper, six natural factors, including relief degree of land surface (RDLS), water resource index (WRI), land cover index (LCI), soil suitability index (SSI), temperature-humidity index (THI), and land surface temperature index (LSTI), were selected to establish the human settlement natural environment index (HNEI) model. With ArcGIS as a tool, the natural suitability of human settlement in five provinces in the lower reaches of the Yangtze river was quantitatively evaluated and analyzed.

(2) The human settlement environment includes five major systems: nature, human, society, residence, and support. There is a strong correlation between each system. The main research object of this study is the natural system, and the GDP Index was used to correct the repeated interference of human factors on various elements. The method is feasible to restore the human ecological system objectively and truly from the angle of nature.

(3) In 2015, the most suitable areas accounted for $22.06 \%$ of the total area. The areas with the highest suitability index were mainly distributed in the plains along the Yangtze river, the northern Zhejiang plain, and the Poyang lake plain. The dominant factors were RDLS and LCI, and the vegetation cover was basically paddy field, which is suitable for living. Suitable areas accounted for $16.85 \%$ of the total area. Some areas were distributed around very suitable areas. In addition, there were parts of paddy fields and dry lands in Jiangsu province, and areas with better hydrothermal conditions in Jiangxi province. The most unsuitable areas accounted for $7.27 \%$ of the total area, and were mainly distributed in southern Zhejiang mountain, Dabie mountain, southern Anhui mountain, Mufu mountain, and Jiuling mountain. Relief degree of land surface (RDLS) was the dominant factor, and soil erosion areas were also mainly distributed in these areas. The unsuitable areas accounted for $20.49 \%$ of the total area. Some areas were distributed around the very unsuitable areas, while others were mainly distributed in southern Jiangxi (the dominant factors were RDLS and THI), western and eastern Zhejiang (the dominant factor was RDLS), and northern Anhui (the dominant factor was THI). The area of medium suitable area accounted for $33.33 \%$, which was widely distributed in each province. Whether these areas will develop into suitable or unsuitable areas in the future depends, to a great extent, on urban development policies and means, which are the key areas that should be paid attention to.

(4) From the perspective of space, the human settlement natural environment index of Shanghai in 2015 was above the average. The best among the rest of the provinces was Jiangsu province, with six cities ranking in the top 15. Zhejiang province, Jiangxi province, and Anhui province had two, two, and four cities, respectively. From the perspective of time, the spatial distribution of the natural suitability of human settlements did not change much from 2005 to 2015, but the overall suitability level was improved for a certain extent, mainly due to the improvement of the temperature-humidity index (THI) and the water resource index (WRI).

Author Contributions: Conceptualization, F.S. and X.H.Y.; methodology, F.S. and F.F.W.; data curation, F.S.; writing, F.S.; visualization, F.S.; project administration, X.H.Y. 
Funding: This research was funded by the Project of the National Key Research Program of China (Nos. 2017YFC0506603, 2016YFC0401305), National Natural Foundation of China (No. 51679007), and the State Key Program of National Natural Science of China (No. 41530635).

Conflicts of Interest: The authors declare no conflict of interest.

\section{References}

1. Aynur, M.; Shi, P.; Zhao, G.J.; Yan, F.; Xue, G.L. Evaluations of living environment suitability of Hotan Prefecture in Xinjiang based on GIS. Arid Land Geogr. 2012, 35, 847-855. (In Chinese)

2. Xie, X.Y.; Zeng, X.; Li, J. Evaluation of Nature Suitability for Human Settlement in Chongqing. Resour. Environ. Yangtze Basin 2014, 23, 1351-1359. (In Chinese)

3. Muhammad, R.; Ruhizal, R.; Mohd, S.; Ahamad, S. Land evaluation suitability for settlement based on soil permeability, topography and geology ten years after tsunami in Banda Aceh, Indonesia. Egypt. J. Remot Sens. Space Sci. 2015, 18, 207-215.

4. Safa, M.; Majed, B.; Doaa, A.H. GIS approach for assessment of land suitability for different land use alternatives in semi arid environment in Jordan: Case study (Al Gadeer Alabyad-Mafraq). Inf. Proc. Agric. 2019, 6, 91-108.

5. Walter, M.R.; Tshesane, M.; Kangethe, M. The strategically located land index support system for human settlements land reform in South Africa. Cities 2017, 60, 91-101.

6. Bagdanavičiūtè, I.; Valiūnas, J. GIS-based land suitability analysis integrating multi-criteria evaluation for the allocation of potential pollution sources. Environ. Earth Sci. 2013, 68, 1797-1812. [CrossRef]

7. Wu, L.Y. Search for the Theory of Science of Human Settlement. Planners 2001, 17, 5-8. (In Chinese)

8. Wu, L.Y. Introduction to Sciences of Human Settlements; China Architecture \& Building Press: Beijing, China, 2001. (In Chinese)

9. Wei, W.; Shi, P.J.; Feng, H.C.; Wang, X.F. Study on the Suitability Evaluation of the Human Settlements Environment in Arid Inland River Basin-A Case Study on the Shiyang River Basin. J. Nat. Resour. 2012, 27, 1940-1950. (In Chinese)

10. Wang, Y.; Jin, C.; Lu, M.Q.; Lu, Y.Q. Assessing the suitability of regional human settlements environment from a different preferences perspective: A case study of Zhejiang Province, China. Habitat Int. 2017, 70, 1-12. [CrossRef]

11. Ma, R.F.; Wang, T.F.; Zhang, W.Z.; Yu, J.H.; Wang, D.; Chen, L.; Jiang, Y.P.; Feng, G.Q. Overview and progress of Chinese geographical human settlement research. J. Geogr. Sci. 2016, 26, 1159-1175. [CrossRef]

12. Deng, M.L.; Zhang, B.; Gao, Y.R.; Yu, B. Assessment of Human Settlements in Prefecture-level Cities of Sichuan Province. Sichuan Environ. 2009, 28, 42-47. (In Chinese)

13. Xia, Y.; Lin, A.W.; Zhu, H.J. Evolvement of Spatial Pattern of Urban Human Settlement Environment Suitability in Yangtze River Delta. Ecol. Econ. 2017, 33, 112-117. (In Chinese)

14. Li, X.; Li, X.X.; Wang, T.; Li, L. Human Settlement Assessment in Yellow River Valley Based on Factor Analysis. Environ. Sci. Technol. 2010, 33, 189-193. (In Chinese)

15. Li, M.; Li, X.M. Application Research on Quality Evaluation of Urban Human Settlements Based on The BP Neural Network Improved by GA. Econ. Geogr. 2007, 32, 99-103. (In Chinese)

16. Zhang, X.R.; Fang, C.L.; Wang, Z.B.; Ma, H.T. Urban Construction Land Suitability Evaluation Based on Improved Multi-criteria Evaluation Based on GIS (MCE-GIS): Case of New Hefei City, China. Chin. Geogr. Sci. 2013, 23, 740-753. [CrossRef]

17. Xu, X.L. China Annual Normalized Difference Vegetation Index Spatial Distribution Data. Resource and Environment Data Cloud Platform, Chinese Academy of Sciences. Available online: http://www.resdc.cn/ DOI/doi.aspx?DOlid=49 (accessed on 2 November 2018). (In Chinese).

18. Xu, X.L. Spatial Distribution Data of Soil Erosion in China. Resource and Environment Data Cloud Platform, Chinese Academy of Sciences. Available online: http://www.resdc.cn/DOI/doi.aspx?DOIid=47 (accessed on 2 November 2018). (In Chinese).

19. Cheng, S.J.; Zhu, Z.L.; Bai, L.B. GIS-based Assessment on Ecological Suitability for Human Settlement-A Case Study in the Central Arid Zone in Ningxia. Arid Zone Res. 2015, 32, 176-183. (In Chinese)

20. Feng, Z.M.; Tang, Y.; Yang, Y.Z.; Zhang, D. Relief degree of land surface and its influence on population distribution in China. J. Geogr. Sci. 2008, 18, 237-246. [CrossRef] 
21. Wang, Y.L.; Qi, P.C.; Li, D.; Ma, X.L. Relief and Suitability Evaluation of the Human Settlements in Shaanxi Province. J. Northwest Normal Univ. 2013, 49, 96-101. (In Chinese)

22. Lang, L.L.; Cheng, W.M.; Zhu, Q.J.; Long, E. A Comparative Analysis of the Multi-criteria DEM Extracted Relief-Taking Fujian Low Mountainous Region as an Example. Geo-Inf. Sci. 2007, 1, 135-136. (In Chinese)

23. Hao, H.M.; Ren, Z.Y. Evaluation of Nature Suitability for Human Settlement in Shaanxi Province Based on Grid Data. Acta Geogr. Sin. 2009, 64, 498-506. (In Chinese)

24. Feng, Z.M.; Yang, Y.Z.; Zhang, D.; Tang, Y. Natural environment suitability for human settlements in China based on GIS. J. Geogr. Sci. 2009, 19, 437-446. [CrossRef]

25. Hao, H.M.; Ren, Z.Y.; Xue, L.; Jiang, Y.F. Quantitative Study on Response of Ecosystem to Land Use/Cover Changes in Yulin Area Based on 3S Technology. Prog. Geogr. 2007, 96, 106-130. (In Chinese)

26. Peng, S.Z.; Yu, K.L.; Li, Z.; Wen, Z.M.; Zhang, C. Integrating potential natural vegetation and habitat suitability into revegetation programs for sustainable ecosystems under future climate change. Agric. For. Meteorol. 2019, 269, 270-284. [CrossRef]

27. State Environmental Protection Administration. Environmental Protection Industry Standard of the People's Republic of China: Technical Criterion for Ecosystem Status Evaluation; China Environmental Science Press: Beijing, China, 2006. (In Chinese)

28. Deng, S.B.; Zhang, Q.N. GIS-Based Evaluation of Natural Suitability of Human Settlement Environment in Guangdong Province. Acta Scientiarum Nat. Univ. Sunyatseni 2014, 53, 127-134. (In Chinese)

29. Li, Y.C.; Liu, C.X.; Zhang, H.; Gao, X. Evaluation on the human settlements environment suitability in the Three Gorges Reservoir Area of Chongqing based on RS and GIS. J. Geogr. Sci. 2011, 21, 346-358. [CrossRef]

30. Qiao, H.J.; Ding, M.J.; Li, L.H.; Chen, Z.P.; Hou, H.Y. Design and Achievement of Soil Erosion Assessment System of Jiangxi Province. Soil Water Conserv. China 2014, 46, 63-69. (In Chinese)

31. Teng, H.F.; Hu, J.; Zhou, Y.; Zhou, L.Q.; Shi, Z. Modelling and mapping soil erosion potential in China. J. Integr. Agric. 2019, 18, 251-264. [CrossRef]

32. Zhao, J.L.; Yang, Z.Q.; Gerard, G. Soil and water conservation measures reduce soil and water losses in China but not down to background levels: Evidence from erosion plot data. Geoderma 2019, 337, 729-741. [CrossRef]

33. Yu, S.; Dai, W.Y. Fujian Province Tour Climate Evaluation. J. Fujian Normal Univ. 2005, 103-106. (In Chinese)

34. Aynur, M.; Wahap, H.; Gulgena, H.; Rebiyam, M. Assessment of residential environment suitability for southern Xinjiang based on GIS. J. Arid Land Resour. Environ. 2012, 26, 11-17. (In Chinese)

35. Halik, W.; Mamat, A.; Dang, J.H.; Deng, B.S.; Tiyip, T. Suitability analysis of human settlement environment within the Tarim Basin in Northwestern China. Quatern. Int. 2013, 311, 175-180. [CrossRef]

36. Bokaie, M.; Zarkesh, M.K.; Arasteh, P.D.; Hosseini, A. Assessment of Urban Heat Island based on the relationship between land surface temperature and Land Use/ Land Cover in Tehran. Sustain. Cities Soc. 2016, 23, 94-104. [CrossRef]

37. Qiao, Z.; Sun, Z.Y.; Sun, X.H.; Xu, X.L.; Yang, J. Prediction and analysis of urban thermal environment risk and its spatio-temporal pattern. Acta Econ. Sin. 2019, 39, 1-10. (In Chinese)

38. Zhu, B.Y.; Li, G.Z.; Liu, C.Y.; Liu, J.F.; Xu, C.H. Evaluation of the natural suitability of human settlements environment in Jilin Province based on RS and GIS. Remot Sens. Land Resour. 2013, 25, 138-142. (In Chinese)

(C) 2019 by the authors. Licensee MDPI, Basel, Switzerland. This article is an open access article distributed under the terms and conditions of the Creative Commons Attribution (CC BY) license (http://creativecommons.org/licenses/by/4.0/). 